\title{
42. A COMPARISON OF THE DENSITY GRADIENTS OF MAIN SEQUENCE STARS OBTAINED BY TWO DIFFERENT METHODS IN DIFFERENT GALACTIC LATITUDES
}

\author{
W. BECKER and R. FENKART \\ Astronomisches Institut der Universität Basel, Binningen, Switzerland
}

The Basel Observatory program of the determination of disc- and halo-density gradients for different intervals of absolute magnitude comprises in addition to Milky Way fields several directions, all pointing to Selected Areas near a plane perpendicular to the galactic equator and passing through the sun and the galactic centre. It was started with SA 51 (Becker, 1965) and continued with Sa 57, 54 and 141 (Fenkart, 1967, 1968, 1969).

The aim of the investigations in the galactic disc is to study stellar clouds and their content of stars of different luminosity, based on the finding that in other galaxies stellar clouds are closely related with spiral structure. In this connection we want above all to check the possibility of applying three-colour photometric methods for this purpose in the simpler case of higher galactic latitudes where interstellar absorption is of no influence.

In each direction we measured photometrically all the 1500 to 2000 stars contained in fields of 0.5 to $2.5 \square^{\circ}$ on Palomar Schmidt plates, and determined their apparent magnitudes in the RGU system with the help of photoelectric scales comprising between 40 and 60 stars per direction most of them observed by Purgathofer (1969). These magnitudes needed no corrections for interstellar absorption, since the twocolour diagrams showed no perceptible reddening for any of the investigated directions.

The blanketing effect shown in the two-colour diagram allowed the statistical separation of the Population II stars from the disc stars, in which we are interested in this context. The two-colour diagram allows also the attribution of statistical values of absolute magnitudes to each disc star.

Thus the density functions could be derived immediately by counting the stars within a given interval of absolute magnitude falling into consecutive intervals of distance modulus.

In the overlapping distance interval these 'photometric' density gradients can be compared with the 'spectral' gradients, that one can obtain by using the spectral type for the determination of the absolute magnitude. The material of the Bergedorfer Spektraldurchmusterung has been used for this purpose.

Our photoelectric scales and the Schmidt plates allowed us to reach limiting magnitudes of 19.5 in G for SA 51,54 and 57 , and $17^{\mathrm{m}} .5$ for SA 141 . So it is evident that the photometric density functions will extend to much farther distances than the spectral ones, since the Bergedorf classification is not sufficiently reliable for our purposes for stars fainter than $13^{\mathrm{m}} .0$ in $\mathrm{B}$.

Therefore the density comparison will be limited to distances within roughly $1 \mathrm{kpc}$ 
or less from the sun, depending on what luminosity interval is used. Finally, in order to be able to compare the photometric and the spectral density functions for the same luminosity groups, we had to establish the relationship between our intervals in absolute magnitude $\left\langle M_{\mathrm{G}}\right\rangle$ and the corresponding intervals in Bergedorf spectral type $<S_{\mathrm{B}}>$. That can be done using the relationships:

Bergedorf- to MK-spectral classification (according to Schmidt-Kaler, 1965),

MK-type to $M_{\mathrm{V}}$, B-V and U-B (according to Schmidt-Kaler, 1965 and Johnson, 1966) and the formula:

$$
G=V+0.93(\mathrm{~B}-\mathrm{V})-0.08(\mathrm{U}-\mathrm{B})
$$

(Steinlin, 1968), as our photometric observations are made in the RGU system.

The density functions are given for the two intervals of absolute magnitude: $3<M_{\mathrm{G}}<5$ and $5<M_{\mathrm{G}}<7$.

The result is shown in Figures 1 and 2 for Selected Areas 54, 57 and 51, 82 respectively. They show in the first line, that the method of three-colour photometry covers distances, which are up to 10 times as large as the distances reached by spectral classification. This is essential for the study of star clouds. Besides, there are pronounced differences in the density values determined by the two different methods. The stars with $M_{\mathrm{G}}$ between 3 and 5 show generally much lower densities if spectral types are used for the determination of absolute magnitude. In the interval between 5 and 7

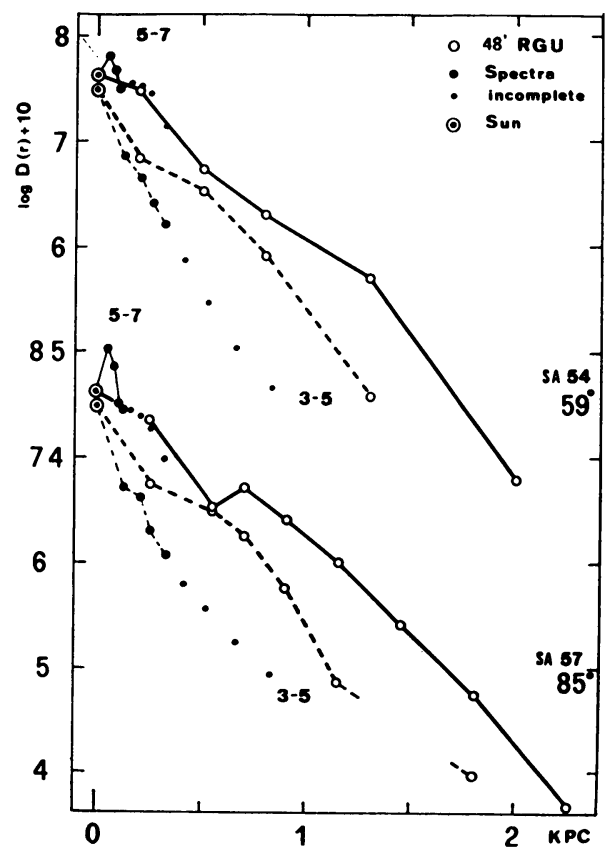

Fig. 1. Density gradients for Selected Areas No. 54 and 57 for intervals of absolute magnitude from 3 to 5 and from 5 to 7 . Strong lines and open circles: RGU photometry; weak lines and points: spectral classification; small points: incomplete data. 


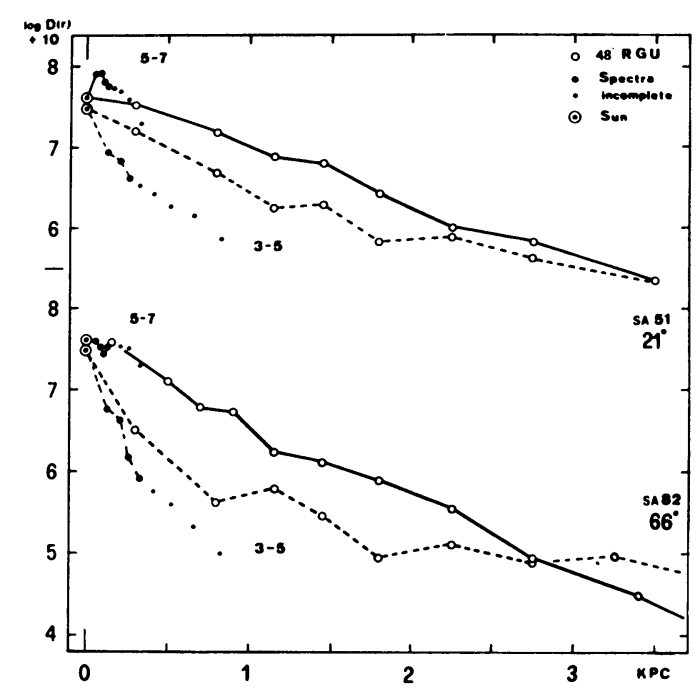

Fig. 2. The same as Figure 1 for Selected Areas No. 51 and 82.

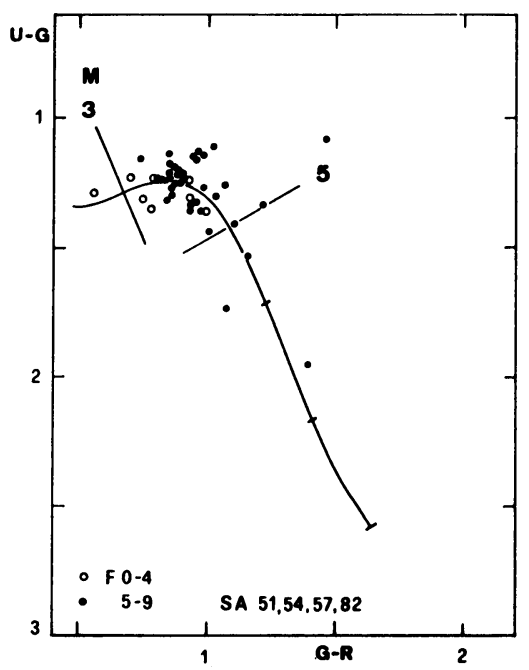

Fig. 3. The distribution of $\mathrm{F}$ stars (Bergedorf classification) in the two-colour diagram. According to their spectral type the stars should have absolute magnitudes between 3 and 5 .

the opposite is the case. The three-colour photometry data match the values in the vicinity of the sun (Gliese, 1957) much better than the spectral type data. We suspect that these discrepancies are caused by systematic effects in classification of $F$ and $G$ type stars in the Bergedorfer Durchmusterung in these fields.

That there exist serious differences in the resulting absolute magnitudes by spectral classification and by three-colour photometry can be seen by plotting into twocolour diagrams those stars in Selected Areas 51, 54, 57 and 82 which are bright enough to be classified in Bergedorf type. 
The result is shown in the Figures 3 and 4, where we have first the stars of Bergedorf-type $\mathrm{F}$, which correspond to the luminosity interval $3<M_{\mathrm{G}}<5$ and then the ones of Bergedorf-type $\mathrm{G}$, corresponding to the luminosity group $5<M_{\mathrm{G}}<7$. Whereas the earlier types fall well in the corresponding luminosity interval defined by threecolour photometry, a considerable fraction of the later ones according to threecolour photometry should evidently be classified earlier, if a reasonable relation between spectral type and absolute magnitude is supposed to exist.

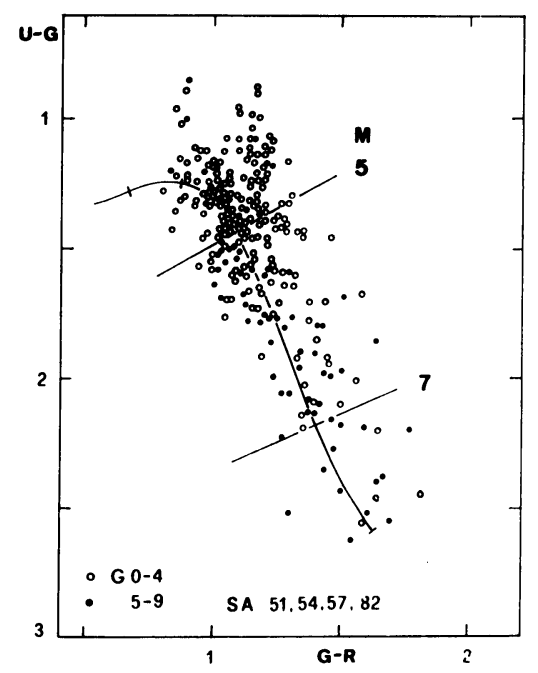

Fig. 4. The distribution of $\mathrm{G}$ stars (Bergedorf classification) in the two-colour diagram. According to their spectral type the stars should have absolute magnitudes between 5 and 7 .

This finding gives at least a qualitative hint, as to where to look for the cause of the observed discrepancies between the spectral and the photometric density gradients. We have to take it into account in the discussion of density gradients determined in lower galactic latitudes.

\section{Acknowledgement}

We thank the Schweizerischer Nationalfonds zur Förderung der Wissenschaftlichen Forschung for financial help.

\section{References}

Becker, W.: 1965, Z. Astrophys. 62, 54.

Fenkart, R.: 1967, Z. Astrophys. 66, 390.

Fenkart, R.: 1968, Z. Astrophys. 68, 87.

Fenkart, R.: 1969, Astron. Astrophys. 3, 228.

Gliese, W.: 1957, Astron. Rechen-Inst. Heidelberg, Mitt. A, No. 8.

Johnson, H. L.: 1966, Ann. Rev. Astron. Astrophys. 4, 193.

Schmidt-Kaler, T.: 1965, Landolt-Börnstein, New Series 1, 284.

Purgathofer, A.: 1969, Lowell Obs. Bull. 147, 98.

Steinlin, U. W.: 1968, Z. Astrophys. 69, 276. 